\title{
PREPARAÇÃO DE MEMBRANAS DE ACETATO DE CELULOSE ORGANOMODIFICADAS PARA ADSORÇÃO DOS ÍONS Cu(II), Cd(II), Mn(II) E Ni(II)
}

\author{
Danielle Goveia e Fabiana Aparecida Lobo \\ Instituto de Química, Universidade Estadual Paulista, Rua Francisco Degni, s/n, 14800-900 Araraquara - SP, Brasil \\ Ubirajara Pereira Rodrigues Filho \\ Instituto de Química de São Carlos, Universidade de São Paulo, CP 780, 13560-970 São Carlos - SP, Brasil \\ Newton Luiz Dias Filho \\ Departamento de Física e Química, Universidade Estadual Paulista, 15385-000 Ilha Solteira - SP, Brasil \\ Leonardo Fernandes Fraceto e André Henrique Rosa* \\ Departamento de Engenharia Ambiental, Universidade Estadual Paulista, Av. Três de Março, 511, 18087-180 Sorocaba - SP, Brasil
}

Recebido em 29/9/09; aceito em 25/1/10; publicado na web em 6/5/10

\begin{abstract}
PREPARATION OF THE ORGAMOMODIFIED CELLULOSE ACETATE MEMBRANES FOR ADSORPTION OF THE IONS $\mathrm{Cu}(\mathrm{II}), \mathrm{Cd}(\mathrm{II}), \mathrm{Mn}$ (II) AND Ni(II). Cellulose acetate polymeric membranes had been prepared by a procedure of two steps, combining the method of phase inversion and the technique of hydrolysis-deposition. The first step was the preparation of the membrane, and together was organomodified with tetraethylortosilicate and 3-aminopropyltrietoxysilane. Parameters that exert influence in the complexation of the metallic ion, as $\mathrm{pH}$, time of complexation, metal concentration, had been studied in laboratory using tests of metal removal. The membranes had presented resistance mechanics and reactivity to cations, being able to be an alternative for the removal, daily pay-concentration or in the study of the lability of metals complexed.
\end{abstract}

Keywords: metals; cellulose acetate; organomodification.

\section{INTRODUÇÃO}

Existem no planeta inúmeras situações de ecossistemas em estresse devido à escassez de água e são vários os casos de disputas existentes entre países que dispõem da mesma fonte de água. A expansão industrial trouxe milhares de novos produtos os quais são aportados aos mananciais. Essas substâncias organossintéticas não degradáveis causam alterações significativas nos ecossistemas aquático e terrestre. Outras espécies químicas que, dependendo da quantidade, contaminam as águas são os metais. Estes representam um grupo especial, pois, não são degradados. ${ }^{1,2} \mathrm{~A}$ ocorrência de metais no ambiente pode ser de origem natural (minérios, rochas sedimentares etc.) ou de origem antrópica como, por exemplo, atividades industriais, turismo, efluentes etc., distribuindo-se no ambiente em razão dos ciclos biogeoquímicos da matéria. $\mathrm{O}$ intemperismo dissolve rochas, podendo transportar metais para rios e lagos, solos adjacentes e oceanos. Os ciclos biológicos incluem a bioacumulação e a biomagnificação, os quais transformam teores normais em concentrações tóxicas, para diferentes espécies da biota e para o próprio homem..$^{3-5}$ A presença de metais no ambiente aquático em concentrações elevadas pode levar à morte de peixes e seres fotossintetizantes, afetar o crescimento de plantas e, quando introduzidos no organismo humano via cadeia alimentar, podem causar doenças, devido a seu efeito cumulativo, e/ ou até a morte. ${ }^{6}$

A presença de metais em águas naturais exerce função determinante no mecanismo biológico de diferentes espécies. Alguns metais podem apresentar toxicidade elevada para determinados organismos, enquanto outros são considerados essenciais.

O termo "metais potencialmente tóxicos" ainda não está bem definido, mas é amplamente reconhecido e utilizado para um grupo de metais e metaloides, que estão associados com poluição e toxicidade $(\mathrm{Pb}, \mathrm{Cd}, \mathrm{Hg}, \mathrm{As}$, e U) e, também, para alguns elementos biologica-

\footnotetext{
*e-mail: ahrosa@ sorocaba.unesp.br
}

mente essenciais em baixas concentrações, como $\mathrm{Co}, \mathrm{Cu}, \mathrm{Mn}, \mathrm{Se}$ e $\mathrm{Zn}$. Nestes casos, tanto a deficiência como o excesso é prejudicial ao organismo. ${ }^{7}$

Muitos procedimentos têm sido desenvolvidos no estudo de metais como, por exemplo, no desenvolvimento de métodos para o monitoramento/remediação de metais em sistemas aquáticos, bem como no conhecimento da forma como os íons se encontram. Como, por exemplo, o desenvolvimento de novos suportes sólidos com esta finalidade. Quando um sólido se encontra em contato com um gás ou uma solução, o gás ou a substância dissolvida na solução (soluto) tende a concentrar-se na superfície do sólido. Esta tendência de acumulação de um composto e/ou íon sobre uma superfície é denominada sorção. ${ }^{8}$ Dentre os diversos materiais suportes que possuem capacidade de sorver íons metálicos, a celulose apresenta propriedades interessantes como material sorvente, principalmente porque permite que sua superfície seja modificada por processos químicos mais simples e de baixo custo conseguindo-se, assim, aumentar a sua capacidade de sorção e/ou troca iônica. ${ }^{9}$ Membranas poliméricas modificadas têm sido desenvolvidas como suporte sólido para uma variedade de aplicações: industriais, ${ }^{10}$ microfiltração e/ou ultrafiltração, ${ }^{11}$ osmose reversa, ${ }^{12}$ separação de gases ${ }^{13}$ e remoção de metais. ${ }^{14}$ Cada aplicação impõe exigências específicas no material e na estrutura da membrana.

Há vários caminhos para se preparar membranas poliméricas, no entanto, a maioria delas é preparada por separação de fases da solução polimérica em duas fases: uma com alta concentração de polímero, e outra com baixa concentração de polímero. A fase concentrada solidifica logo após a separação da fase e dá forma à membrana. ${ }^{15}$ Assim, o objetivo deste trabalho foi preparar membranas de acetato de celulose e propor a organomodificação utilizando reagentes contendo o grupo amino, que são reativos aos metais de interesse, para posterior utilização na remoção e/ou pré-concentração de metais, ou no estudo da labilidade de metais complexados por substâncias húmicas em sistemas aquáticos. 


\section{PARTE EXPERIMENTAL}

\section{Reagentes}

Todos os reagentes utilizados foram de pureza analítica. Ácidos e soluções alcalinas necessárias para ajuste de $\mathrm{pH}$ foram preparadas por diluição de $30 \%$ de acido clorídrico (ultrapuro, Merck AG, Alemanha) ou hidróxido de sódio mono-hidratado (ultrapuro, Merck AG, Alemanha) em água de alta pureza (resistividade $18,2 \mathrm{M} \Omega / \mathrm{cm}$ a $25^{\circ} \mathrm{C}$, sistema Milli-Q plus, Millipore, USA). Acetato de celulose (conteúdo de 39,8\% de acetila, Sigma-Aldrich), tetraetilortossilicato (98\%, Aldrich, EUA) e 3-aminopropiltrietóxissilano $\left(\mathrm{C}_{9} \mathrm{H}_{23} \mathrm{NO}_{3} \mathrm{Si}-99 \%\right.$, Aldrich, EUA) foram usados para preparação e modificação da membrana. A resina XAD 8 (Serva Feinbiochemica, Germany) utilizada para a isolação das SHA foi pré-purificada por sucessivos enxágues com $0,5 \mathrm{~mol} \mathrm{~L}^{-1} \mathrm{HCl}, 0,5 \mathrm{~mol} \mathrm{~L}^{-1} \mathrm{NaOH}$ e metanol (24 h, cada). As soluções de metais foram preparadas por diluição da solução estoque (1000 mg L ${ }^{-1}$; Normex, Carlo Erba). A limpeza da vidraria foi feita utilizando vapor de ácido nítrico concentrado, segundo procedimento proposto por Tschöpel e colaboradores ${ }^{16}{ }^{16}$ seguido de armazenamento por imersão em ácido nítrico $10 \%$ (v/v). Antes da utilização a vidraria foi enxaguada exaustivamente com água desionizada.

\section{Preparação das membranas}

A membrana polimérica foi preparada pelo método de inversão de fase utilizando-se a seguinte percentagem em massa dos reagentes: $11 \%$ de acetato celulose, $23 \%$ de ácido acético, $43 \%$ de acetona e $23 \%$ de água desionizada, conforme descrito por Rodrigues-Filho e colaboradores. ${ }^{17}$ Foi adicionado $1 \%(\mathrm{~m} / \mathrm{m})$ de plastificante di-isodecil adipato (DIDA cedido pela Petrom - Petroquímica Mogi das Cruzes - SP, Brasil) à massa total de solução polimérica. As quantidades de reagentes utilizadas para $30 \mathrm{~g}$ de solução polimérica foram: 3,3 $\mathrm{g}$ de acetato de celulose, $6,6 \mathrm{~mL}$ de ácido acético, 16,3 $\mathrm{mL}$ de acetona e $6,9 \mathrm{~mL}$ de água e adição de $0,33 \mathrm{~mL}$ de plastificante. Esta quantidade de solução polimérica é suficiente para preparar três membranas poliméricas e apresenta boa homogeneização.

A solução permaneceu sob agitação com agitador magnético por 8 h, para a homogeneização completa. Em seguida, foi transferida para uma placa de vidro, usada como suporte, utilizando-se dois fios finos de $0,33 \mathrm{~mm}$ para o espalhamento. Os fios foram enrolados nas bordas da placa de vidro e deslizando-se um bastão de vidro sobre eles foi possível controlar a espessura das membranas. O tempo de espalhamento da solução polimérica foi de $35 \mathrm{~s}$ para a evaporação do solvente. A placa de vidro revestida com a solução polimérica foi imersa em um banho com água desionizada e retirada após desprendimento da membrana. ${ }^{17}$ As membranas foram cortadas em círculos de $47 \mathrm{~mm}$ para utilização nos experimentos. As membranas preparadas pelo método de inversão de fase foram imersas em solução de hidróxido de sódio $\left(0,25 \mathrm{~mol} \mathrm{~L}^{-1}\right)$ a $40{ }^{\circ} \mathrm{C}$ por $1 \mathrm{~min}$ para desacetilação das membranas.

Para a organomodificação, as membranas foram deixadas em etanol por no mínimo 10 min, imersas em solução etanólica 2,2\% (v/v) de tetraetilortossilicato (TEOS) por 4 min e, em seguida, em solução de ácido nítrico $\left(10^{-3} \mathrm{~mol} \mathrm{~L}^{-1}\right)$ para hidrólise do TEOS e novamente imersas em etanol por $10 \mathrm{~min}$. O ancoramento do grupo amino foi feito pela imersão em solução etanólica de 3-aminopropiltrietoxissilano (3-APTS) $\left(10^{-3} \mathrm{~mol} \mathrm{~L}^{-1}\right)$ por $4 \mathrm{~min}$. As membranas foram armazenadas em água.

Estudo dos parâmetros que influenciam na adsorção de cátions metálicos pelas membranas organomodificadas

Influência do pH e do tempo na adsorção de cátions metálicos

Para estudo da complexação de cátions metálicos pela membrana polimérica em função do $\mathrm{pH}$ utilizaram-se $250 \mathrm{~mL}$ de solução padrão multielementar de íons metálicos (Cu(II), Cd(II), $\mathrm{Ni}$ (II) e $\mathrm{Mn}(\mathrm{II})$ ) $1,0 \mathrm{mg} \mathrm{L}^{-1}$, com diferentes valores de $\mathrm{pH}(3,0-7,0)$. Cada solução foi transferida para um béquer de $500 \mathrm{~mL}$, nos quais se colocaram uma membrana organomodificada. Sob agitação mecânica foram retiradas alíquotas de 4,0 mL em função do tempo (0-1440 min), que foram acidificadas e as concentrações dos íons determinadas por espectrometria de absorção atômica. As concentrações dos íons complexados pela membrana polimérica em função do tempo foram obtidas pela diferença entre as concentrações iniciais dos metais $(\mathrm{t}$ $=0)$ e as concentrações determinadas nas alíquotas retiradas nos diferentes intervalos de tempo.

\section{Estudo das isotermas de adsorção em função da concentração de metal e do $\mathrm{pH}$ da solução}

O estudo da complexação de cátions metálicos pela membrana em função da concentração do metal foi feito utilizando-se o método de batelada, à temperatura ambiente. Cada membrana organomodificada foi colocada em béquer contendo $250 \mathrm{~mL}$ de solução padrão multielementar de íons metálicos (Cu(II), Cd(II), Ni(II) e Mn(II)) em diferentes concentrações $\left(0,25\right.$ - 3,0 mg L $\left.\mathrm{m}^{-1}\right)$ e pH 5,0. Retirou-se uma alíquota no tempo inicial $(\mathrm{t}=0)$ e outra após $12 \mathrm{~h}$ de agitação mecânica. As alíquotas foram acidificadas e as concentrações dos íons determinadas por espectrometria de absorção atômica. A concentração do metal sorvido foi determinada pela diferença entre as concentrações iniciais e finais das alíquotas coletadas. O tempo necessário para o equilíbrio foi obtido a partir dos resultados da adsorção em função do tempo.

\section{Aplicação das membranas organomodificadas}

A labilidade de $\mathrm{Cu}$ (II), complexados por substâncias húmicas aquáticas (SHA), foi investigada por estudos de troca iônica em batelada. As SHA utilizadas no estudo da labilidade de $\mathrm{Cu}$ (II) foram isoladas de amostras de água coletadas em afluentes dos rios Itapanhaú e Ribeira de Iguape (municípios de Bertioga e Iguape, respectivamente, SP, Brasil), por cromatografia adsortiva em coluna, utilizando resina Amberlite XAD 8. Este procedimento foi proposto por Thurman e Malcolm ${ }^{18}$ e descrito pela International Humic Substances Society.

Para a obtenção dos complexos entre íons Cu e SHA (Cu-SHA) foram preparadas soluções contendo concentrações variadas de SHA e íons $\mathrm{Cu}(\mathrm{II})$, com pHs ajustados. Os volumes foram ajustados para $250 \mathrm{~mL}$ com água deionizada e as soluções armazenadas durante $24 \mathrm{~h}$.

Os experimentos foram feitos em batelada e ficaram sob agitação durante $8 \mathrm{~h}$; utilizou-se um excesso de tempo para manter um equilíbrio entre as ligações CU-SHA e Cu-Membrana. Para cada solução foi colocada uma membrana polimérica organomodificada e retirada uma alíquota no tempo zero (concentração inicial de íons $\mathrm{Cu}$ ) e uma alíquota após este período (concentração final de íons $\mathrm{Cu}$ presentes na solução) e os metais determinados por espectrometria de absorção atômica em chama. Assim a concentração dos íons $\mathrm{Cu}$ complexados à membrana polimérica foi determinada pela diferença $\mathrm{Cu}_{\text {inicial }}-\mathrm{Cu}_{\text {final }}=\mathrm{Cu}_{\text {complexado }}$.

\section{Determinações de cátions metálicos}

Cobre, manganês, cádmio e níquel foram determinados por espectrometria de absorção atômica com atomização por chama (Varian, AA240FS), utilizando-se as condições operacionais descritas no manual do fabricante. Utilizou-se curva de calibração multielementar preparada a partir da diluição de soluções estoque $\left(1000 \mathrm{mg} \mathrm{L}^{-1}\right)$.

\section{RESULTADOS E DISCUSSÃO}

A membrana polimérica foi preparada utilizando-se a técnica de inversão de fase. Nesta técnica ocorre uma separação de fases, uma 
fase rica e uma pobre em polímero que foi conseguida pela técnica de imersão-precipitação. A solução polimérica é imersa em um banho de não solvente (neste caso, água destilada) para a precipitação do polímero, onde no processo de transferência de massa ocorre a troca de solvente/não solvente.

A fase polimérica rica forma a matriz da membrana, enquanto a fase pobre em polímero origina os poros. Dependendo de algumas condições de preparação, como evaporação, espessura da membrana e composição, a estrutura da membrana obtida varia de simétrica e densa a assimétrica. As membranas assimétricas são caracterizadas pela presença de duas fases ao longo da membrana. Na interface com o substrato é formada uma fase densa, fina e nanoporosa chamada de filtrante. Na interface com a suspensão polimérica é formada uma camada densa e espessa com poros que podem atingir algumas centenas de nanometros. A espessura e porosidade da camada filtrante determinam o desempenho da separação, enquanto a subcamada fornece o suporte mecânico. Diversos são os parâmetros de processo e composição na preparação da membrana assimétrica que podem afetar na morfologia da membrana e no seu desempenho. ${ }^{19}$

As membranas poliméricas podem se apresentar quebradiças, o que poderia gerar problemas futuros referentes ao manuseio e à sua aplicação, sendo necessário acrescentar um plastificante para melhorar a resistência mecânica. Foram feitos testes com diversos plastificantes, sendo que o dilsodecil adipato (DIDA) apresentou melhores resultados com concentrações baixas. DIDA é um plastificante monomérico de massa molar média, com baixa viscosidade e pouca volatilidade, o que o qualifica para aplicações em que são exigidas alta resistência mecânica e excepcional flexibilidade, principalmente à temperatura ambiente.

Depois de prontas, as membranas passaram pelas etapas de organomodificação:

$1^{a}$ etapa - a hidrólise catalisada por base é utilizada para a desacetilação do acetato de celulose e proporciona uma maior adesão do dióxido de silício na superfície da membrana. Esta etapa tem a função de aumentar o número de sítios ativos, $\mathrm{C}_{\text {polímero }}-\mathrm{OH}$, para sorção de sílica e, consequentemente, a maior adsorção de aminossilanos;

$2^{\mathrm{a}}$ etapa - a formação do híbrido de acetato de celulose e sílica por meio da adsorção de tetraetilortossilicato e posterior hidrólise em meio ácido com solução de ácido nítrico para formar a sílica. A formação do dióxido de silício se dá pela reação de oxalação entre as moléculas de TEOS hidrolisadas, ou seja, pela reação entre várias moléculas através dos grupos silanois formados na hidrólise deste reagente. A ligação $\mathrm{Si}-\mathrm{OH}$ é mais reativa que a $\mathrm{C}-\mathrm{OH}$, a carga negativa no oxigênio ligado ao silício é maior, tornando um nucleófilo forte e favorecendo a substituição;

$3^{\text {a }}$ etapa - a imobilização do grupo amino na membrana polimérica preparada (celulose+TEOS) ocorreu através da reação da celulose com o 3-aminopropiltrietóxissilano (3-APTS) como agente de sililação. A incorporação deste grupo tornou a membrana mais reativa a cátions metálicos, devido à presença dos grupos reativos disponíveis (Figura 1). A superfície da membrana atua como uma boa base de Lewis, devido à presença do par de elétrons livre presente no átomo de nitrogênio.

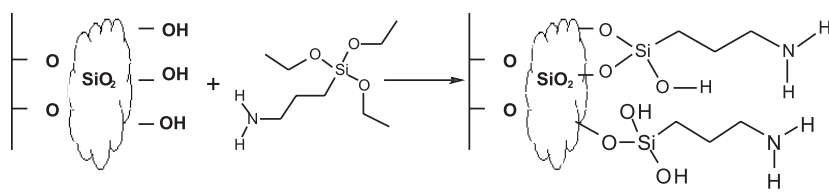

Figura 1. Incorporação do grupo amino utilizando como precursor o 3-aminopropiltrietoxissilano

A evidência de que ocorreu a funcionalização da membrana polimérica foi comprovada por sua capacidade de reter íons $\mathrm{Cu}(\mathrm{II})$. A superfície da membrana polimérica organomodificada adquiriu uma coloração fracamente azulada após a imersão na solução aquosa de sulfato de cobre 1,0 mol L-1 água desionizada, indicando a ocorrência da organomodificação e adsorção dos íons $\mathrm{Cu}(\mathrm{II})$. O mesmo não ocorreu quando se realizou o teste com as membranas não modificadas.

\section{Estudo dos parâmetros que influenciam na adsorção de cátions} metálicos pelas membranas celulósicas e organomodificadas

A Figura 2 ilustra a complexação dos cátions metálicos em $\mathrm{pH}$ 5,0 , geralmente o $\mathrm{pH}$ encontrado para águas naturais, pela membrana polimérica em função do tempo. Neste $\mathrm{pH}$ o diagrama de Pourbaix indica que os íons $\mathrm{Ni}$ estão presentes na forma de $\left[\mathrm{Ni}\left(\mathrm{OH}_{2}\right)_{6}\right]^{2+}{ }_{\text {aq }}$ como espécies em solução aquosa. ${ }^{20} \mathrm{O}$ mesmo acontece com os demais $\left[\mathrm{M}\left(\mathrm{OH}_{2}\right)_{6}\right]^{2+}{ }_{\text {(aq) }}{ }^{21}$ A partir dos valores de remoção pode-se observar a seguinte ordem crescente da capacidade de retenção pela membrana: $\mathrm{Ni}(\mathrm{II})<\mathrm{Cd}(\mathrm{II})<\mathrm{Mn}(\mathrm{II})<\mathrm{Cu}$ (II)

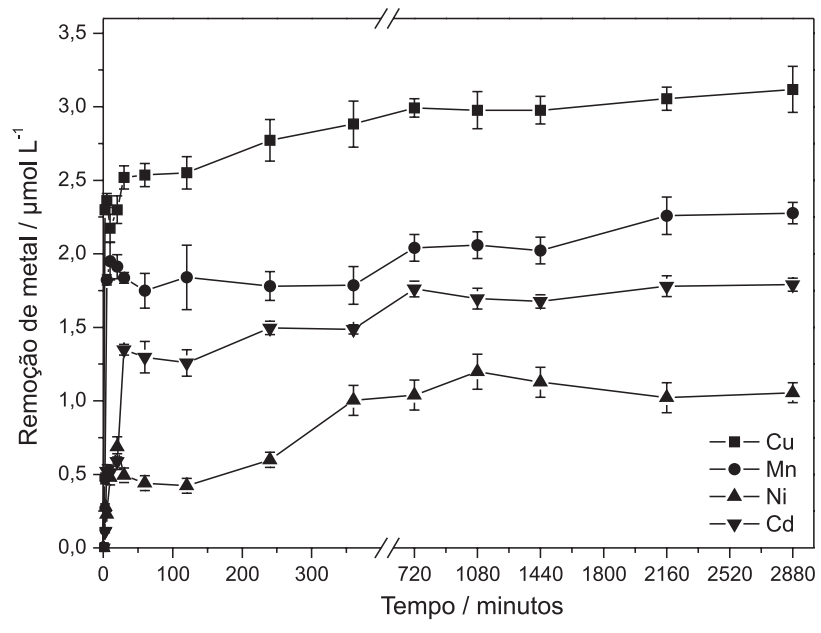

Figura 2. Cinética da remoção de cátions metálicos (Cu(II), Cd(II), Mn(II) e Ni(II)) a partir de solução aquosa pela membrana polimérica modificada com 3-APTS/SiO ${ }_{2}$. Condições: $250 \mathrm{~mL}$ de solução aquosa contendo [Cu]=16; $[\mathrm{Mn}]=18 ;[\mathrm{Ni}]=17$ e $[\mathrm{Cd}]=9 \mu \mathrm{mol} \mathrm{L}^{-1}$, um disco de membrana com $47 \mathrm{~mm}$ de diâmetro, $p H$ 5,0, $t=48 \mathrm{~h}$, sob agitação mecânica

Para $\mathrm{Cd}$ e $\mathrm{Cu}$ observa-se que há adsorção praticamente instantânea. No Cd ocorre um patamar e no $\mathrm{Cu}$, não. Isto deve indicar que para o Cd há um mecanismo de sorção diferente, provavelmente envolvendo duas espécies químicas diferentes na superfície dos poros. Pode ser que o $\mathrm{Cu}$ forme logo $\mathrm{Cu}(3 \text {-APTS })_{2}$ e o $\mathrm{Cd}$ forme primeiro $\mathrm{Cd}(3-\mathrm{APTS})$ e, em seguida, $\mathrm{Cd}(3-\mathrm{APTS})_{2}$ ou podem ocorrer ligações entre o $\mathrm{Cd}$ e os grupos $\mathrm{C}-\mathrm{OH}$ do acetato de celulose e/ou grupos $\mathrm{Si}-\mathrm{OH}$ da sílica.

Os íons Ni apresentam um mínimo em cerca de 90 min, para depois atingir um patamar em torno de $400 \mathrm{~min}$. Este comportamento indica que se podem ter duas espécies diferentes sendo formadas no interior da membrana com o tempo, uma lábil no início da curva e outra inerte, podendo estar associada a diferentes geometrias, formase primeiro uma espécie monocoordenada lábil que se decompõe para depois se formar uma espécie bidentada.

O tratamento cinético descrito por Burba e van den Bergh foi aplicado para estimar o tempo necessário para o equilíbrio, pela relação da concentração do íon no equilíbrio com a concentração do mesmo em cada alíquota. ${ }^{22}$ Os tempos de equilíbrio indicam uma cinética lenta para os íon $\mathrm{Mn}(\mathrm{II})$ e $\mathrm{Ni}(\mathrm{II})$ (305 e $435 \mathrm{~min}$ ) e mais rápidas para $\mathrm{Cu}(\mathrm{II})$ e $\mathrm{Cd}(\mathrm{II})$ (94 e $185 \mathrm{~min}$ ). 
Na literatura ${ }^{23}$ encontra-se a seguinte série de tempo de vida para a troca da água $\left(\tau_{-} \mathrm{H}_{2} \mathrm{O}\right)$ para os cátions $\mathrm{Cu}<\mathrm{Cd}<\mathrm{Mn}<\mathrm{Ni}$, ou seja, o níquel tem a cinética de saída da água mais lenta. Este parâmetro é importante, pois estes íons devem ter o mesmo mecanismo de complexação pela membrana, ou seja, $\mathrm{I}_{\mathrm{D}}$, intermediário dissociativo. Neste mecanismo, uma boa parte da constante de velocidade do processo é dominada pela velocidade de saída da água. A série é obedecida de acordo com os tempos de equilíbrio encontrados.

A Figura 3 compara a capacidade de retenção dos cátions metálicos em diferentes valores de $\mathrm{pH}$ após $24 \mathrm{~h}$ de equilíbrio. Observa-se que a membrana polimérica apresentou capacidades de complexação que variaram de 104 a $198 \mu \mathrm{g} \mathrm{L}^{-1}$ para o $\mathrm{Cu}(\mathrm{II})$, com valores maiores em pH 5,0. Já o Mn(II) apresentou valores de retenção aproximadamente constantes $\left(110 \mu \mathrm{g} \mathrm{L}^{-1}\right)$ e independentes do $\mathrm{pH}$, com um pequeno aumento em $\mathrm{pH} 4,0\left(150 \mu \mathrm{g} \mathrm{L}^{-1}\right)$. $\mathrm{Ni}$ (II) apresentou valores crescentes entre $\mathrm{pH} 3,0$ e 6,0, variando de 32 a $94 \mu \mathrm{g} \mathrm{L} \mathrm{L}^{-1}$ de remoção e, em pH 7,0, apresentou um decréscimo para cerca de 20 $\mu \mathrm{g} \mathrm{L}^{-1}$ de íon metálico retido. Já o íon Cd(II) apresentou valores de retenção com uma variação de 50 a $200 \mu \mathrm{g} \mathrm{L}{ }^{-1}$, com um máximo em pH 5,0. Esta influência provavelmente está associada à formação de hidroxo-complexos em detrimento dos aquo-complexos formados pelos cátions metálicos em função da variação do $\mathrm{pH}$.

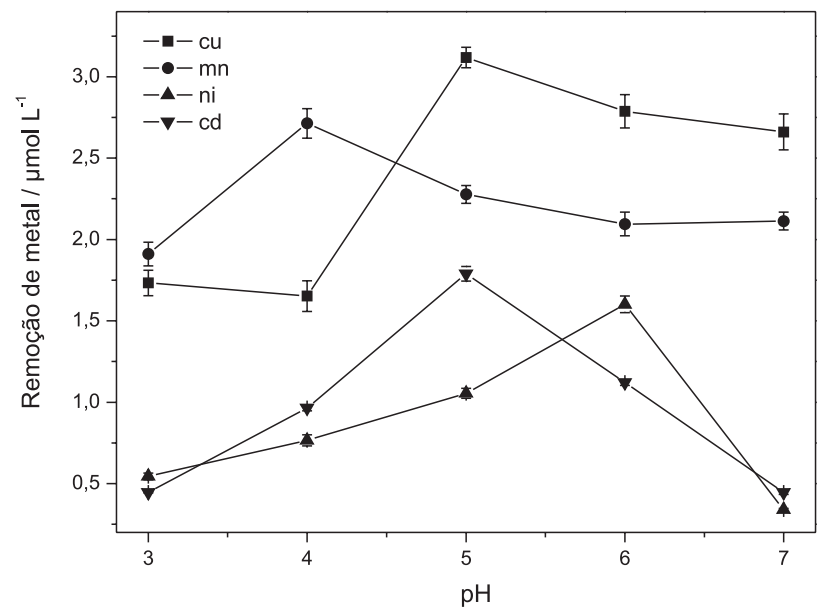

Figura 3. Remoção de cátions metálicos a partir de solução aquosa pela membrana polimérica modificada com 3-APTS/SiO ${ }_{2}$. Condições: $250 \mathrm{~mL}$ de solução aquosa contendo $[\mathrm{Cu}]=16 ;[\mathrm{Mn}]=18 ;[\mathrm{Ni}]=17$ e $[\mathrm{Cd}]=9 \mathrm{\mu mol}$ $L^{-1}$, um disco de membrana com $47 \mathrm{~mm}$ de diâmetro, $\mathrm{pH}$ 3,0-7,0, tempo de complexação de 24 h, sob agitação mecânica

Em baixos valores de $\mathrm{pH}$, provavelmente ocorre a protonação do grupo amino, dificultando a complexação dos íons metálicos em função da competição dos prótons pelos sítios básicos de Lewis, $-\mathrm{NH}_{2} \mathrm{e}-\mathrm{OH}$, e provocando a repulsão de espécies iônicas por exclusão Donnan pela membrana polimérica carregada positivamente. Na literatura, superfícies modificadas com reagentes contendo o grupo terminal $\mathrm{NH}_{2}$ apresentaram protonação dos grupos amino abaixo de $\mathrm{pH} 4,5 .{ }^{24}$

Os resultados de retenção referentes à membrana polimérica não seguiram a ordem de estabilidade termodinâmica da série de Irving-Willian, pois o Mn está invertido com o $\mathrm{Ni}$ ( $\mathrm{pH} 5,0)$. Esta inversão indica que não se tem sempre a mesma espécie química na sorção dos diferentes tipos de metais na membrana. A série de Irving-Williams mostra que a estabilidade dos complexos metálicos segue a variação do raio iônico dos íons e a energia de estabilização do campo cristalino, ou seja, o preenchimento dos orbitais $t_{2 g} e_{\mathrm{g}}$. A série apresenta a seguinte ordem de estabilidade em relação ao íon metálico: $\mathrm{Mn}(\mathrm{II})<\mathrm{Ni}(\mathrm{II})<\mathrm{Cu}(\mathrm{II})$.
A capacidade de adsorção foi avaliada pelo comportamento de isotermas para os cátions em estudo. Quantitativamente, a adsorção pode ser avaliada por parâmetros como $\mathrm{C}_{\mathrm{s}}$, concentração do soluto na solução, quando estabelece o equilíbrio de adsorção e $\mathrm{N}_{\mathrm{f}}$, número de mols de metal adsorvido, para $1 \mathrm{~g}$ da membrana polimérica. Do ponto de vista experimental, o valor de $\mathrm{N}_{\mathrm{f}}$ foi obtido do número inicial de moles de cátion $\left(\mathrm{n}_{\mathrm{i}}\right)$ adicionado ao sistema e este no equilíbrio $\left(\mathrm{n}_{\mathrm{s}}\right)$ para a massa de membrana polimérica em gramas $(\mathrm{m})$, aplicando a seguinte expressão: $\mathrm{N}_{\mathrm{f}}=\left(\mathrm{n}_{\mathrm{i}}-\mathrm{n}_{\mathrm{s}}\right) / \mathrm{m}$. As isotermas de adsorção são mostradas na Figura 4.

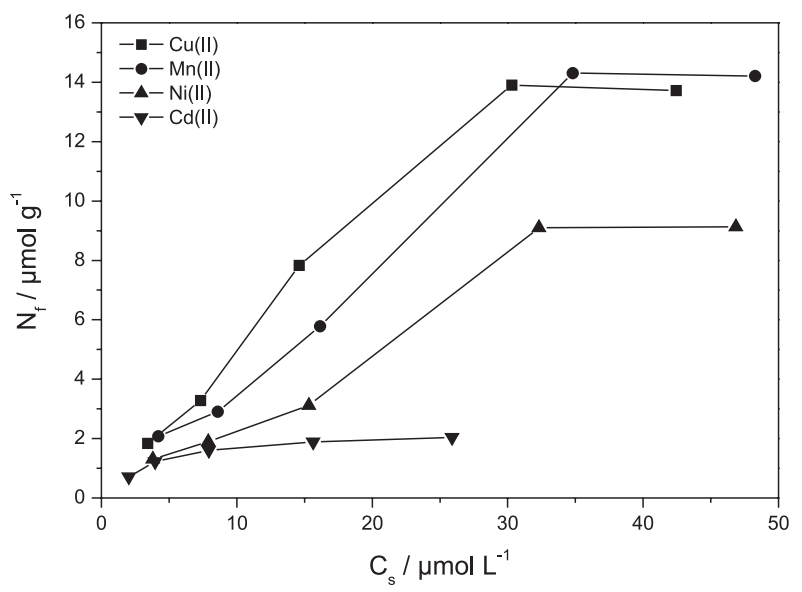

Figura 4. Isotermas de adsorção de cátions metálicos pela membrana modificada no tempo de equilíbrio. Condições: $250 \mathrm{~mL}$ de solução multielementar (contendo concentrações variadas de $\mathrm{Cu}(\mathrm{II}), \mathrm{Mn}(\mathrm{II}), \mathrm{Ni}(\mathrm{II})$ e Cd(II), uma membrana modificada, pH 5,0 agitação mecânica, tempo de complexação de 12 he temperatura ambiente

Com o aumento da concentração do metal, a quantidade do material adsorvido na superfície sólida tende a um valor constante. Nessa situação, os sítios de adsorção na superfície do material sorvente encontram-se completamente saturados e a quantidade de substância sorvida não depende mais da concentração do metal.

Langmuir, em 1918, a fim de explicar fenômenos de adsorção, considerou que as moléculas adsorvidas se fixavam na superfície do sorvente em sítios definidos e localizados, onde cada sítio permitiria apenas uma entidade adsorvida e a energia correspondente seria a mesma em todos os sítios da superfície. Esse tipo de adsorção ocorre sempre que uma quantidade adsorvida atinge um valor máximo a uma determinada concentração e permanece constante. O processo completo de adsorção pode ser descrito quantitativamente por meio de isotermas de adsorção, que à temperatura constante, fornecem dados relacionados à quantidade adsorvida $\left(\mathrm{N}_{\mathrm{f}}\right)$ e à concentração do soluto $\left(\mathrm{C}_{\mathrm{s}}\right)$, depois de estabelecido o equilíbrio. ${ }^{25}$

A linearização das isotermas de adsorção foi feita com a aplicação da Equação de Langmuir:

$$
\frac{C_{S}}{N_{f}}=\frac{C_{s}}{N_{s}}+\frac{1}{N_{S} k}
$$

onde $\mathrm{C}_{\mathrm{s}}$ é a concentração em solução no equilíbrio $\left(\mathrm{mmol} \mathrm{L}^{-1}\right), \mathrm{N}_{\mathrm{f}}$ foi definido anteriormente $\left(\mathrm{mmol} \mathrm{g}^{-1}\right), \mathrm{N}_{\mathrm{s}}$ é a quantidade máxima de soluto adsorvido por grama de superfície sorvente $\left(\mathrm{mmol} \mathrm{g}^{-1}\right)$, que depende do número de sítios de adsorção e $\mathrm{k}$ é a medida de intensidade de adsorção, diretamente relacionada com uma constante de equilíbrio $\left(\mathrm{mol} \mathrm{L}^{-1}\right)$. A linearização das isotermas de adsorção foi plotada como apresentado na Figura 5 e os valores das constantes $\mathrm{k}$ e $\mathrm{N}_{\mathrm{s}}$ foram calculados para cada interação superfície-cátion. 


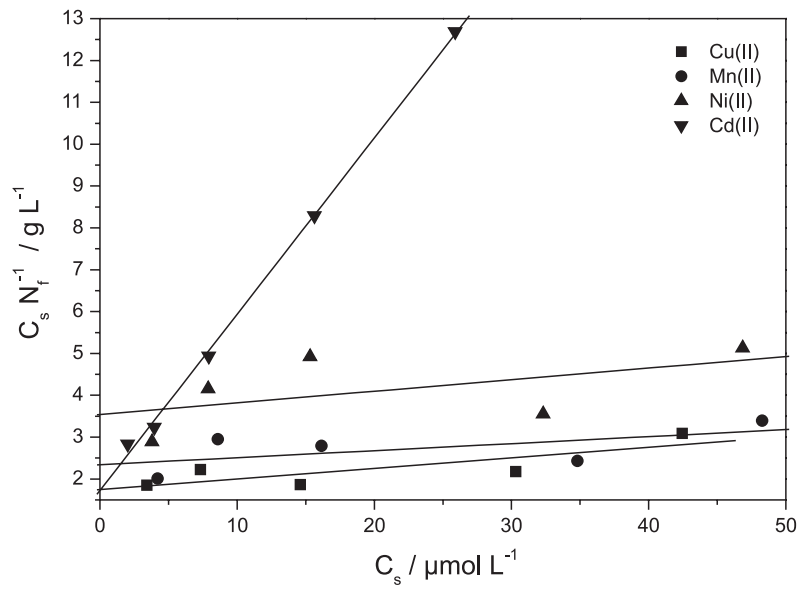

Figura 5. Isotermas linearizadas de adsorção de cátions metálicos pela membrana polimérica organomodificada no tempo de equilíbrio em $\mathrm{pH}$ 5,0

De forma geral, foi possível fazer a linearização das curvas para as espécies $\mathrm{Cu}, \mathrm{Mn}$ e $\mathrm{Cd}$. As capacidades de adsorção determinadas para cada metal na condição de saturação foram $\mathrm{Mn}(\mathrm{II})=59,66 ; \mathrm{Cu}$ (II) $=39,68$ e Cd (II) $=2,37 \mu \mathrm{mol} \mathrm{g} ~^{-1}$. Estes resultados apresentam similaridades para experimentos feitos utilizando suportes sólidos à base de celulose. ${ }^{26}$ Os valores de intensidade de adsorção $(\mathrm{k})$ foram $\mathrm{Mn}(\mathrm{II})=7,15 ; \mathrm{Cu}(\mathrm{II})=14,40$ e Cd (II) $=2,44 \times 10^{2}$. Comparandose esses valores, verifica-se que os complexos Cd-Membrana são mais estáveis.

Considerando as baixas concentrações de metais originalmente presentes em sistemas aquáticos, a membrana organomodificada apresenta-se como um suporte sólido adequado para a complexação com metais nesses sistemas.

\section{Aplicação das membranas organomodificadas}

Em águas naturais, vários ligantes têm a capacidade de reduzir os efeitos tóxicos de metais. Isso tem sido atribuído à complexação dos metais pelos ligantes presentes na água e geralmente essa propriedade é referida como a "capacidade complexante da água". Esta parece estar muito associada com a matéria orgânica aquática. As substâncias húmicas aquáticas (SHA) representam a principal classe de complexantes naturais que compõem a matéria orgânica, influenciando nas reações associadas à complexação, transporte, (bio)disponibilidade e labilidade de espécies metálicas no ambiente. ${ }^{5}$

Vários métodos têm sido utilizados para se obter informações sobre a capacidade complexante das SHA por íons metálicos e o cobre tem sido amplamente usado nesses estudos, em razão de sua característica de formar complexos estáveis com vários ligantes de ocorrência natural na água. ${ }^{5}$ Portanto, este foi o íon metálico escolhido para se verificar a competição entre os sítios complexantes do suporte sólido desenvolvido e as SHA. Inicialmente, há espécies formadas entre os íons $\mathrm{Cu}$ e a SHA (SHA-Cu) e, a partir do momento em que se introduz a membrana no sistema, os íons $\mathrm{Cu}$ ligados fraca/moderadamente às moléculas de SHA ao entrarem em contato com os sítios complexantes da membrana, provavelmente, formarão complexos Cu-Membrana; estes metais são denominados metais lábeis. O aumento da concentração de SHA aumenta a probabilidade de ocorrerem ligações fortes entre SHA e íons $\mathrm{Cu}$ diminuindo a labilidade das espécies metálicas; este comportamento pode ser verificado na Figura 6. Isto ocorre, provavelmente, devido ao fato da macromolécula húmica apresentar elevado grau de condensação em solução concentrada de SHA, tornando os metais menos acessíveis ao contato com os sítios complexantes da membrana polimérica. ${ }^{27}$

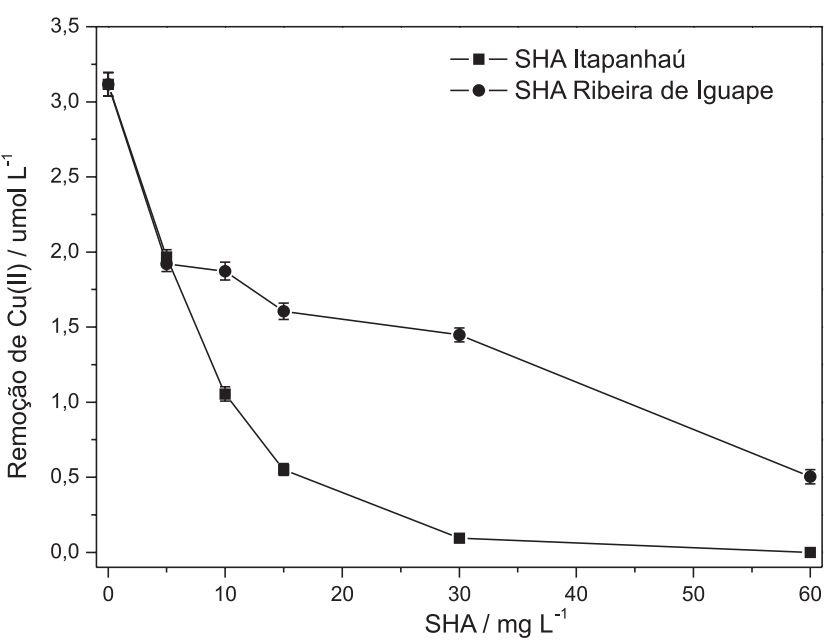

Figura 6. Concentração de Cu(II) complexado pela membrana organomodificada na ausência e presença de SHA-Itapanhaú e SHA-Ribeira de Iguape. Condições: $250 \mathrm{~mL}$ de solução contendo $1000 \mu \mathrm{g} \mathrm{L}^{-1}$ de Cu(II), SHA-Itapanhaú e SHA-Ribeira de Iguape (0-60 $\left.\mathrm{mg} \mathrm{L}^{-1}\right)$, uma membrana organomodificada, pH 5,0, agitação mecânica e tempo de complexação de $24 \mathrm{~h}$

Observa-se também que nas concentrações acima de $15 \mathrm{mg} \mathrm{L}^{-1}$, a remoção de íons cobre pela membrana foi menor na presença de SHA-Itapanhaú do que na presença de SHA-Ribeira de Iguape, ou seja, os íons $\mathrm{Cu}$ apresentaram maior afinidade pelos grupos presentes na SHA-Itapanhaú. Frequentemente as interações metálicas são interpretadas com base no princípio de dureza e maciez de ácidos e bases, proposto por Pearson. ${ }^{28}$ Este propôs uma teoria de afinidade, uma espécie "macia" em geral tem grande raio atômico, baixa carga efetiva nuclear e alta polarizabilidade, enquanto que uma espécie "dura" possui características opostas. Enquanto os íons metálicos são considerados ácidos de Lewis, seus contra-íons são átomos ligantes com características de dureza e maciez. No contexto, estes ligantes estão representados pelas SHA, pelos grupos reativos da membrana e pela água. Embora possam existir algumas exceções, a regra geral enuncia que ácidos duros se ligam a bases duras, enquanto ácidos macios preferem bases macias. Os íons $\mathrm{Cu}(\mathrm{I})$ e $\mathrm{Cu}$ (II) possuem características de ácido mole e intermediário, respectivamente, que preferencialmente interagem com bases de mesma classificação, formando complexos mais estáveis. Em estudos anteriores, Rosa e colaboradores caracterizaram as amostras de SHA extraídas dos mananciais em estudo por análise de espectroscopia de ressonância magnética nuclear ${ }^{13} \mathrm{C} .{ }^{30}$ As amostras de SHA-Itapanhaú apresentaram maior concentração de grupos carboxílicos e fenólicos em sua estrutura que as amostras de SHA-Ribeira de Iguape; estes grupos apresentam características de base mole, o que justifica a pequena diferença de afinidade dos íons $\mathrm{Cu}$ pela SHA-Itapanhaú.

A competição dos íons $\mathrm{Cu}$ (II) pelos sítios complexantes das SHA e da membrana organomodificada em função do aumento do pH é apresentada na Figura 7. Na presença de SHA a concentração de $\mathrm{Cu}$ (II) complexado na membrana polimérica continuou aumentando após o pH 5,0, o que não ocorreu na sua ausência. Na estrutura das SHA, em razão da ionização de grupos de ácidos carboxílicos, há predominância de cargas negativas de ocorrência natural dependendo do $\mathrm{pH}$ do meio. ${ }^{30}$ Desta forma, os sítios complexantes das SHA podem deixar os íons $\mathrm{Cu}(\mathrm{II})$ livres para formar complexos com a membrana polimérica, devido à ação tamponante das SHA.

Apesar das diferenças estruturais das SHA, o comportamento de competição por íons $\mathrm{Cu}$ (II) apresentou certa similaridade, mostrando que a labilidade de metais em sistemas aquáticos não é apenas altamente dependente das condições do corpo d'agua e características 


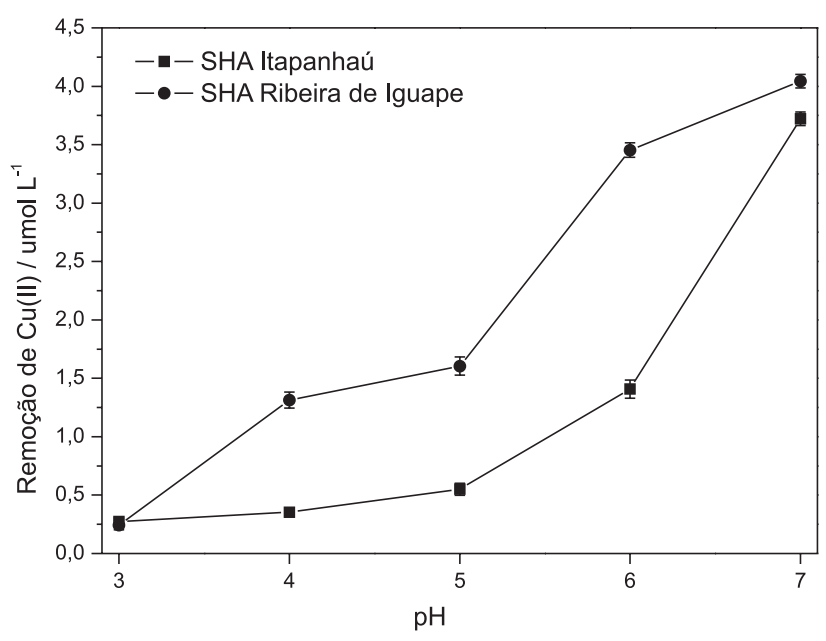

Figura 7. Concentração de Cu(II) complexado pela membrana organomodificada na presença de SHA em diferentes $\mathrm{pHs}$. Condições: $250 \mathrm{~mL}$ de solução contendo $1000 \mu \mathrm{g} \mathrm{L}{ }^{-1}$ dos cátions Cu(II), SHA-Itapanhaú e SHA-Ribeira de Iguape (15 $\mathrm{mg} \mathrm{L}^{-1}$ ), uma membrana organomodificada, $p H$ 3,0-5,0, agitação mecânica e tempo de complexação de $24 \mathrm{~h}$

das SHA, mas também do grupo reativo que irá interagir/competir com as espécies metálicas presentes no ambiente.

\section{CONCLUSÕES}

Membranas de acetato de celulose foram preparadas pelo método de inversão de fases e permitiram o ancoramento de grupos amino, utilizando tetraetilortossilicato (TEOS) e 3-aminopropiltrietóxissilano (3-APTS). A incorporação do plastificante deu resistência mecânica à membrana. $\mathrm{O}$ suporte sólido mostrou-se eficiente na remoção de cátions metálicos (Cu(II), Cd(II), Ni(II) e Mn(II)) em solução aquosa pela formação de complexos entre os sítios complexantes da membrana e os cátions metálicos. O estudo da labilidade de $\mathrm{Cu}$ (II) em sistemas aquáticos demonstrou que a remoção do metal pela membrana diminui com o aumento da concentração de SHA, onde o equilíbrio é deslocado na direção do favorecimento das espécies SHA-metal.

\section{MATERIAL SUPLEMENTAR}

Encontra-se disponível em http://quimicanova.sbq.org.br, a Figura $1 \mathrm{~S}$, na forma de arquivo .PDF, com acesso livre.

\section{REFERÊNCIAS}

1. Alloway, B. J.; Heavy metal in soils, $2^{\text {nd }}$ ed., Wiley: New York, 1993.

2. Rainbow, P. S.; Environ. Int. 2007, 33, 576.

3. Vicente-Martorell, J. J.; Galindo-Riaño, M. D.; García-Vargas, M., Granado-Castro, M. D.; J. Hazard. Mater. 2009, 162, 823.

4. Wang, W-X.; Rainbow, P. S.; Comp. Biochem. Physiol. 2008, 148, 315.

5. Rocha, J. C.; Rosa, A. H.; Substâncias húmicas aquáticas: interações com espécies metálicas, Ed. UNESP: São Paulo, 2003.

6. Chapman, P. M.; Allen, H. E.; Godtfredsen, K.; Z Graggen, M. N.; Environ. Sci. Technol. 1996, 30, 448.
7. Florence, T. M.; Morrison, G. M.; Stauber, J. L. ; Sci. Total Environ. 1992, 125, 1.

8. Ciola, R.; Fundamentos de catálise, Ed. EDUSP: São Paulo, 1981; Dias Filho, N. L.; do Carmo, D. R.; Rosa, A. H.; Sep. Sci. Technol. 2006, 41, 733.

9. O'Connell, D. W.; Birkinshaw, C.; O’Dwyer, T. F.; Bioresour. Technol. 2008, 99, 6709.

10. Ravanchi, M. T.; Kaghazchi, T.; Kargari, A.; Desalination 2009, 235, 199.

11. Albrecht, W.; Santoso, F.; Lützow, K.; Weigel, T.; Schomäcker, R.; Lendlein, A.; J. Membr. Sci. 2007, 292, 145; Yeu, S.; Lunn, J. D.; Rangel, H. M.; Shantz, D. F.; J. Membr. Sci. 2009, 327, 108.

12. Chen, G.; Li, S.; Zhang, X.; Zhang, S.; J. Membr. Sci. 2008, 310, 102.

13. Li, Y.; Chung, T. S. ; J. Membr. Sci. 2008, 308, 128.

14. Melo, J. C. P. de; Silva Filho, E. C. da; Santana, S. A. A.; Airoldi, C.; Colloids Surf., A 2009, 346, 138; Dey, R. K.; Airoldi, C.; J. Hazard. Mater. 2008, 156, 95; Dias Filho, N. L.; do Carmo, D. R.; Rosa, A. H.; Electrochim. Acta 2006, 52, 965.

15. van de Witte, P.; Dijkstra, P. J.; van den Berg, J. W. A.; Feijen, F.; J. Membr. Sci. 1996, 117, 1.

16. Tschöpel, P.; Kotz, L.; Shulz, W.; Veber, M.; Tölg, G.; Fresenius J. Anal. Chem. 1980, 302, 1.

17. Rodrigues-Filho, U. P.; Gushikem, Y.; Gonçalves, M. C.; Cachichi, R. C.; Chem. Mater. 1996, 8, 1375.

18. Thurman, E. M.; Malcolm, R. L.; Environ. Sci. Technol. 1981, 15, 463.

19. Altinkaya, A. S.; Yenal, H.; Ozbas, B.; J. Membr. Sci. 2004, 249, 163.

20. Beverskog, B.; Puigdomenech, I.; Corros. Sci. 1997, 39, 969.

21. Beverskoga, B.; Puigdomenech, I.; J. Electrochem. Soc. 1997, 144, 3746; Park, C. K.; Kim, U. S.; J. Korean Chem. Soc. 1985, 29, 637.

22. Burba, P.; van den Bergh, J.; Anal. Bioanal. Chem. 2004, 378, 1637.

23. Shriver, D. F.; Atkins, P. W.; Langford, C. H.; Inorganic Chemistry, $2^{\text {nd }}$ ed., Oxford University Press: Oxford, 1994; Burgess, J.; Metal ions in solutions, Ellis Horwood: Chichester, 1978.

24. Magalhães, J. L.; Moreira, L. M.; Rodrigues-Filho, U. P.; Giz, M. J.; Pereira-da-Silva, M. A.; Landers, R.; Vinhas, R. C. G.; Nascente, P. A. P.; Surf. Interface Anal. 2002, 33, 293; Fiorilli, S.; Rivolo, P.; Descrovi, E.; Ricciardi, C.; Pasquardini, L.; Lunelli, L.; Vanzetti, L.; Pederzolli, C.; Onida, B.; Garrone, E.; J. Colloid Interface Sci. 2008, 321, 235; Allen, G. C.; Sorbello, F.; Altavilla, C.; Castorina, A.; Filiberto, E.; Thin Solid Films 2005, 483, 306.

25. Britto, P. F.; Dissertação de Mestrado, Universidade Estadual Paulista, Brasil, 2005; Grillo, R.; Melo, N. F. S.; Moraes, C. M.; Lima, R.; Menezes, C. M. S.; Ferreira, E. I.; Rosa, A. H.; Fraceto, L. F.; J. Pharm. Biomed. Anal. 2008, 47, 297.

26. Rosa, A. H.; Goveia, D.; Bellin, I. C.; Lessa, S. S.; Dias Filho, N. L.; Padilha, P. M.; Anal. Bioanal. Chem. 2006, 386, 2153.

27. Ghosh, K.; Schnitzer, M.; Soil Sci.. 1980, 129, 266.

28. Pearson, R. G.; J. Am. Chem. Soc. 1963, 85, 3533.

29. Rosa, A. H.; Bellin, I. C.; Goveia, D.; Oliveira, L. C.; Lourenço, R. W.; Dias Filho, N. L.; Burba, P.; Anal. Chim. Acta 2006, 567, 152.

30. Rocha, J. C.; Sargentini Jr., E.; Zara, L. F.; Rosa, A. H.; Santos, A.; Burba, P.; Talanta 2003, 61, 699. 


\section{PREPARAÇÃO DE MEMBRANAS DE ACETATO DE CELULOSE ORGANOMODIFICADAS PARA ADSORÇÃO DOS ÍONS Cu(II), Cd(II), Mn(II) E Ni(II)}

\section{Danielle Goveia e Fabiana Aparecida Lobo}

Instituto de Química, Universidade Estadual Paulista, Rua Francisco Degni, s/n, 14800-900 Araraquara - SP, Brasil

Ubirajara Pereira Rodrigues Filho

Instituto de Química de São Carlos, Universidade de São Paulo, CP 780, 13560-970 São Carlos - SP, Brasil

Newton Luiz Dias Filho

Departamento de Física e Química, Universidade Estadual Paulista, 15385-000 Ilha Solteira - SP, Brasil

Leonardo Fernandes Fraceto e André Henrique Rosa*

Departamento de Engenharia Ambiental, Universidade Estadual Paulista, Av. Três de Março, 511, 18087-180 Sorocaba - SP, Brasil

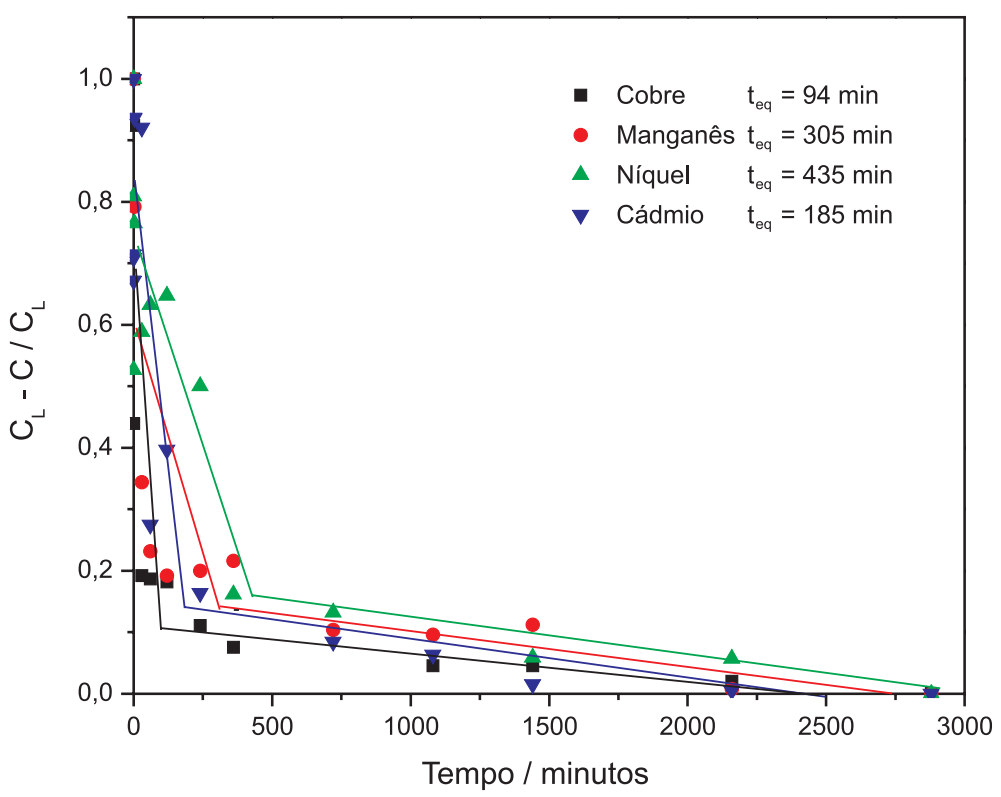

Figura 1S. Estudo cinético das interações entre os cátions metálicos e a membrana polimérica modificada. Condições: $250 \mathrm{~mL}$ de solução contendo 1,0 mg L-1 dos cátions $\mathrm{Cu}(\mathrm{II}), \mathrm{Cd}(\mathrm{II}), \mathrm{Mn}(\mathrm{II})$ e Ni(II), uma membrana polimérica modificada, pH 5,0, agitação mecânica e tempo de complexação de 0-48 $\mathrm{h}$ 